\title{
Castration-resistant prostate cancer: a strategy to enhance response to androgen deprivation
}

\author{
Yuri Stanevsky ${ }^{1}$, Alexander Tsivian ${ }^{1}$ and Matvey Tsivian $^{2}$ \\ Asian Journal of Andrology (2013) 15, 709-710; doi:10.1038/aja.2013.96; Published online: 29 July 2013
}

A new research study evaluated the mechanisms of prostate cancer $(\mathrm{PCa})$ resistance to androgen deprivation therapy (ADT) and progression to castrate-resistant disease. Using a coclinical approach, the investigators were able to identify key genetic determinants of ADT resistance, gain insight into the molecular pathways that play a key role in this transition and propose a potential management strategy to overcome ADT resistance in select cancers. This represents a step forward towards personalized medicine guided by specific molecular markers of prostate cancer enabling more effective therapies targeted towards altered metabolic pathways.

Androgen deprivation therapy (ADT) remains the main management option for advanced and metastatic prostate cancer (PCa). Although the majority of patients promptly respond to ADT, most will eventually progress to castrate-resistant $\mathrm{PCa}$ (CRPC), characterized by cancer growth despite castrate levels of androgens. To date, limited options exist for the treatment of CRCP that is generally considered deadly. ${ }^{1}$ Lunardi et al. ${ }^{2}$ investigated the mechanisms of ADT resistance using a coclinical approach whereby they used both murine models and clinical human data to comprehensively assess the pathways involved in CRPC development.

The mouse models have shown differential response to castration based on the specific genetic make-up of the tumor model. Similarly, in the human specimens analyses, the same genetic determinants were found to be associated both with clinical response to

${ }^{1}$ Department of Urologic Surgery, The E. Wolfson Medical Center, Holon and Sackler school of Medicine, Tel Aviv University, Tel Aviv 53000, Israel and ${ }^{2}$ Division of Urology, Department of Surgery, Duke University Medical Center, Durham, NC 27710, USA

Correspondence: Dr M Tsivian (matvey.tsivian@ duke.edu)
ADT and progression to CRPC. Lunardi et al. ${ }^{2}$ have also detailed the molecular events that accompany progression to CRPC, suggesting important pathways that could be acted upon. These accompanying molecular features the authors have identified could be responsible for the inhibition of apoptosis and lack of proliferation arrest. Key roles in this phenomenon are played by the Xaf1 pathway, which can be inhibited by embelin. Interestingly, when Xafl pathway is inhibited with embelin, androgen deprivation suppresses tumor growth in vivo.

In the study of Lunardi et al., ${ }^{2}$ the authors suggest that tumors exhibiting deregulation of Xaf1 and SRD5al pathways are prone to developing castration resistance. In these patients, however, the inhibition of these pathways (by embelin and dutasteride) may result in dramatically improved patient response to ADT.

This study of genetic characteristics of PCa progression poses several interesting and important points for discussion. First, the 'coclinical' approach ${ }^{2}$ the authors used for this study is comprehensive and integrates both preclinical and clinical data, thus enhancing the applicability and translation of the results into clinical practice. Such an approach allows for research hypotheses to be tested against the clinical complexity of a disease and to link the available animal models of cancer to the diverse patient population with their diverse tumor make-up. We believe that such an approach offers tremendous advantages for translational research.

Second, prostate cancer is an extremely heterogeneous disease, both from the clinical characteristics, and, not surprisingly, its genetic and phenotypic combination. The strength of this study is that the findings from the murine models were readily confirmed in human PCa specimens allowing for drawing a parallel with clinical applicability. In fact, alterations of Pten, TP53 and Zbtb7a are common in advanced prostate cancer.,

Third, the authors further suggest that in a subset of $\mathrm{PCa}$ tumors, characterized by impairment of Xaf1-Xiap and SRD5A1 pathways, the combination of ADT with specific inhibitors - embelin and dutasterideresults in sensitization of these cancers to the effects of ADT, thereby dramatically increasing the efficiency of this treatment approach in patients that would otherwise be on the pathway of rapid progression to CRPC. Despite the importance of this finding, several questions arise with regards to the applicability of these results to the clinical practice. ${ }^{5}$

With the advances in technology and genetic testing capabilities, it is likely that a simple and inexpensive procedure could be available clinically to test for these, and other genetic markers. It would be interesting to know whether such testing would be possible on biopsy cores as opposed to known PCa in a radical prostatectomy specimen. Moreover, the authors analyzed specimens with Gleason 6-7 PCa, which is not necessarily the salient characteristic of aggressive, presumably deadly PCa. In light of concern for overtreatment for localized $\mathrm{PCa},{ }^{6}$ it is unclear whether these specimens would be representative of current and, most importantly, future PCa. Another question is would the inclusion of embelin as a promoter of apoptosis increase the effectiveness of ADT and other treatment modalities (even local ablative techniques) or in managing tumors that do not express alterations of the culprit pathways.

Individualized management of cancer is the goal clinicians strive for and progress is being made in defining the optimal treatment for a given patient. ${ }^{7}$ It is clear that 'one size fits all' approaches have gaps. ${ }^{8}$ Characterization of individual factors associated with treatment responses by means of understanding of the molecular pathways leading to the 
clinical outcome, will enable the community to benefit not only from novel therapeutic regimens but would also enable us to identify the best suited management that is tailored to the individual patient. We believe this future is not too far away, although some challenges are evident. ${ }^{5}$

In summary, this is an important study identifying not only genetic predictors of response to ADT and the mechanisms of resistance, but also suggests a differential treatment strategy based on the genetic make-up of the tumor. This is an important step towards understanding the mechanisms of PCa progression, and, potentially, a step towards a novel therapeutic approach for aggressive PCa.

Karantanos T, Corn PG, Thompson TC. Prostate cancer progression after androgen deprivation therapy: mechanisms of castrate resistance and novel therapeutic approaches. Oncogene; e-pub ahead of print 10 June 2013; doi:10.1038/onc.2013.206.

2 Lunardi A, Ala U, Epping MT, Salmena L, Clohessy JG et al. A co-clinical approach identifies mechanisms and potential therapies for androgen deprivation resistance in prostate cancer. Nat Genet 2013; 45: 747-55.

3 Liu W, Xie CC, Thomas CY, Kim ST, Lindberg J et al. Genetic markers associated with early cancer-specific mortality following prostatectomy. Cancer 2013; 119: 2405-12.
4 Wang G, Lunardi A, Zhang J, Chen Z, Ala U et al. Zbtb7a suppresses prostate cancer through repression of a Sox9-dependent pathway for cellular senescence bypass and tumor invasion. Nat Genet 2013; 45: 739-46.

5 Lam YW. Scientific challenges and implementation barriers to translation of pharmacogenomics in clinical practice. ISRN Pharmacol 2013; 2013: 641089.

6 Wilt TJ, Brawer MK, Jones KM, Barry MJ, Aronson WJ et al. Radical prostatectomy versus observation for localized prostate cancer. N Engl J Med 2012; 367: 203-13.

7 Bitting RL, Armstrong AJ. Potential predictive biomarkers for individualizing treatment for men with castration-resistant prostate cancer. Cancer J 2013; 19: 25-33.

8 Ayres BE, Sooriakumaran P. Future prospects: who is the patient who still dies of prostate cancer following local treatment of high-risk prostate cancer? Can we prolong their lives using modern treatment approaches? Curr Opin Urol 2013; 23: 372-6. 